\section{Guiding light}

Bioluminescence is a useful tool in modern biological experiments. For example, the brilliant light emitted from the reaction catalyzed by luciferase, often used as a reporter for transcription activation. Luciferases bind and modify a small organic compound,

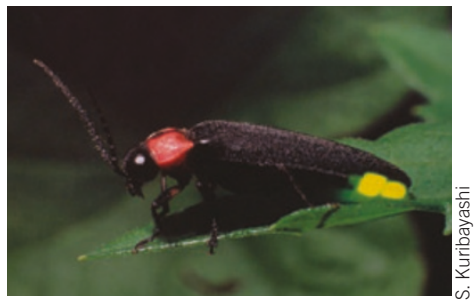
luciferin, at the expense of ATP. This reaction produces a high-energy reaction intermediate that breaks down to generate the light-emitting moiety oxyluciferin. The light emitted by firefly luciferases ranges from yellow-green to yellow-orange, but a single mutation in the enzyme can shift the emission color to red. How the enzymes tune the color of the emitted light was not understood. To address this question, Kato and colleagues have determined the crystal structure of the wild-type luciferase (emitting yellow-green light) from a Japanese firefly in complex with an analog of the high-energy reaction intermediate. They have also solved the structure of a mutant luciferase emitting red light in complex with the same compound. A comparison of these structures reveals that the hydrophobicity and the rigidity of the protein surrounding oxyluciferin are important for the color of the emitted light. The structural observation is consistent with mutagenesis data and sequence conservation. Understanding how the color of the emitted light alters with changes in the luciferase sequence may allow for the engineering of new molecules useful for biological experiments. (Nature 440, 372-376, 2006)

$H P F$

\section{Self-destruction and DNA repair}

In response to UV damage or stalled replication, PCNA, a DNAreplication processivity factor, is activated by monoubiquitination and localized to the replication fork. PCNA then recruits a translesion synthesis (TLS) polymerase such as poln to help bypass the DNA lesion and rescue the stalled fork. PCNA can recruit both error-free and errorprone TLS polymerases, so its activity must be tightly regulated. The deubiquitinating enzyme USP1 has previously been shown to negatively regulate components of the Fanconi anemia DNA repair pathway. Now, D'Andrea and colleagues show that USP1 is also active in regulating TLS repair. They have found that overexpression of USP1 results in decreased levels of PCNA monoubiquitination, whereas short interfering RNAmediated knockdown of USP1 has the opposite effect. The former observations require a catalytically active USP1 and a particular lysine on PCNA, indicating that USP1 deubiquitinates PCNA at a specific site. UV exposure triggers a two-step USP1 degradation that begins with USP1 cleavage and is followed by proteasomal degradation of the $\mathrm{N}$-terminal fragment. Additional experiments suggest that USP1 bears a ubiquitinlike domain, and the authors identify an internal cleavage site found directly downstream of this putative domain, indicating a self-cleavage mechanism similar to that found in polyubiquitin-chain cleavage and processing. In all, the studies suggest that active PCNA levels, and therefore TLS polymerase recruitment, are tightly regulated by the deubiquitinating activity of USP1 and that UV exposure relieves this negative regulation. The molecular signal that triggers USP1 autocleavage activity remains to be determined. (Nat. Cell Biol., advance online publication 12 March 2006, doi:10.1038/ncb1378)

$M M$

Research highlights written by Anna Barnett, Hwa-ping Feng,

Boyana Konforti and Michelle Montoya.

\section{Imidazole to the rescue}

Deleterious mutations in the active sites of some enzymes can be complemented by small compounds that functionally substitute for the mutant's missing side chain. Such small-molecule rescue quickly and reversibly switches on a formerly inactive enzyme. This process may hold promise for treating genetic diseases, and it is particularly useful in analyzing signaling pathways such as the network of kinases that regulates cell division and cancer. In a recent study, Cole and colleagues show that imidazole restores the tyrosine kinase function of Src, the first-discovered proto-oncoprotein, after the mutation of a conserved catalytic arginine. This rescue occurs in vivo as well as in vitro and leads to cellular phenotypes associated with the cancer-causing viral Src kinase. The authors exploit the rescue of mutant Src by imidazole to search for proteins phosphorylated by Src, identifying several new potential substrates, including the cytoskeletal signaling protein CrkL. The authors further demonstrate effects of the growth factors EGF, PDGF and FGF on CrkL phosphorylation and MAP kinase activation by Src, helping to delineate pathways that can lead to normal replication or cancer. (Science 311, 1293-1297, 2006)

\section{Balancing act at the ends}

Telomeres are nucleoprotein structures that cap the ends of linear chromosomes in eukaryotic cells. They maintain the integrity of chromosomes, protect them from degradation and from end-to-end fusion with other chromosomes. The DNA component of telomeres usually begins with a doublestranded DNA region consisting of repeated sequences and ends in TG-rich single-stranded (ss) DNA. Telomeres are also substrates for the enzyme telomerase, which is responsible for replicating the ends of chromosomes in cell division. In yeast, telomerase recruitment is mediated by the Cdc13-Ten 1-Stn 1 complex and the Yku70-Yku80 heterodimer, and these proteins function in telomere capping as well as telomere elongation. Defects in telomere capping generate large amounts of ssDNA at telomeres, initiate a robust DNA damage checkpoint response and lead to genome instability. To identify novel regulators of telomere structure in budding yeast, Durocher, Lydall and colleagues screened for suppressors of the temperature-sensitive $c d c 13-1$ allele. They found five new cdc13-1 suppressors and focused on Cgi121, which encodes a highly conserved protein. They show that Cgi121 is part of a conserved protein complex, KEOPS, which contains a protein kinase, Bud32. Deletion of Ggi121 suppresses $c d c 13-1$ by reducing the levels of SsDNA in the cell. Cells lacking any one of the KEOPS components show stable telomere shortening, indicating that these components have a positive role in telomere elongation. More specifically, Bud32 and Cgi121 act both as positive regulators of telomere elongation and as promoters of telomere uncapping. Although it is unclear whether these two different aspects of telomere regulation by the KEOPS complex are the result of a single biochemical activity, the authors propose a model in which the KEOPS complex regulates access to the chromosome ends. Understanding how telomere end protection is structurally and functionally linked to telomere elongation will require further work. (Cell 124, 1155-1168, 2006) 